\title{
Prognostic Relevance of Survivin in Pancreatic Endocrine Tumors
}

\author{
Sara Ekeblad • Margareta Halin Lejonklou • \\ Peter Stålberg • Britt Skogseid
}

Published online: 17 November 2011

(C) The Author(s) 2011. This article is published with open access at Springerlink.com

\begin{abstract}
Background Better prognostic markers are needed for pancreatic endocrine tumors. Survivin is an apoptosis inhibitor that is suggested to have a negative prognostic impact in several tumor types. Contradictory data exist, especially regarding the significance of a nuclear versus cytoplasmic location of survivin. The prognostic relevance of nuclear and cytoplasmic survivin expression in pancreatic endocrine tumors-controlled for the tumor Ki-67 index, World Health Organization classification, and TNM stage-was investigated.

Methods A total of 111 patients treated at a tertiary referral center were retrospectively evaluated. Clinical data were gathered from medical records. Immunohistochemistry for survivin and $\mathrm{Ki}-67$ was performed on paraffinembedded tissue. Univariate and multivariate Cox analyses were performed.

Results Patients with tumors that had $<5 \%$ survivin-positive nuclei had a mean survival of 225 months [95\% confidence interval (CI) 168-281]. The corresponding figure for patients with 5 to $50 \%$ survivin-positive tumor cell nuclei was 101 months [95\% CI 61-140; hazard ratio (HR) 2.4; $P<0.01)$ and with $>50 \%$ survivin-positive nuclei 47 months $(95 \%$ CI 24-71; HR 4.9; $P<0.001)$. Nuclear
\end{abstract}

Sara Ekeblad and Margareta Halin Lejonklou contributed equally to this study.

S. Ekeblad · M. H. Lejonklou · B. Skogseid ( $\square)$

Department of Medical Sciences, Uppsala University,

75185 Uppsala, Sweden

e-mail: britt.skogseid@medsci.uu.se

P. Stålberg

Department of Surgical Sciences, Uppsala University,

Uppsala, Sweden survivin expression in $>50 \%$ of the tumor cells was an independent marker of a poor prognosis (HR 5.7; $P<0.01$ ). Cytoplasmic survivin was not a significant prognostic factor in the multivariate analysis (HR 0.94; $P=0.90$ ).

Conclusions High expression of nuclear survivin is a significant marker of a poor prognosis in patients with a pancreatic endocrine tumor.

\section{Introduction}

Pancreatic endocrine neoplasms are rare. They are generally less aggressive than pancreatic exocrine cancers but can often be metastatic at diagnosis. The prognosis varies considerably; some patients live for years with liver metastases, whereas others rapidly succumb to progressive disease. Better prognostic criteria are needed for more accurate prognostic guidance for the individual patient.

Data regarding survival and prognostic factors exist mainly from smaller series of patients, and studies often include a mix of tumor identities, such as bronchial and midgut carcinoids and pancreatic endocrine tumors. Some studies have included only patients who have undergone surgery, omitting patients with a heavy tumor burden at diagnosis, possibly creating a selection bias. A median survival of 38 to 104 months $[1,2]$ and a 5-year survival rate of 40 to $60 \%[3,4]$ have been reported. A World Health Organization (WHO) classification system [5] is used to divide tumors into three groups: well-differentiated neuroendocrine tumors, well-differentiated neuroendocrine carcinomas, and poorly differentiated neuroendocrine carcinomas. Ki-67 has been demonstrated to have prognostic value using a cutoff of 2,5 , or $10 \%$ [1, 4, 6-10].

In 2006, a new tumor-node-metastasis (TNM) system for the classification of pancreatic endocrine tumors was 
proposed [11]. Fischer et al. [12], in a study of 118 patients who had undergone surgery, concluded that the classification was of prognostic value. Independently, we made similar observations in an evaluation of 324 patients [10].

Survivin, also known as BIRC5, is a member of the family of apoptosis inhibitors. In the cytoplasm, survivin suppresses apoptosis [13-15], and in the nucleus it takes part in the regulation of cell division $[16,17]$. Survivin is abundantly expressed in the fetus but rarely in adult tissues. Most cancers express the antigen [18], which has aroused great interest as a potential drug target for cancer treatment. Several approaches are being investigated (e.g., immunotherapy, gene therapy, molecular survivin antagonists), and clinical trials are ongoing $[19,20]$.

Several reports have examined the prognostic relevance of survivin expression in various tumor types. A high expression of nuclear survivin has been suggested to correlate with a poor outcome, although results have been somewhat contradictory. Some authors have reported a negative prognostic impact of high nuclear survivin expression in lung cancer and hepatocellular carcinoma $[21,22]$, and others have proposed the opposite in gastric cancers and breast cancer [23, 24]. Cytoplasmic survivin has been suggested to be an important predictor of poor outcome in patients with pancreatic exocrine cancer [25].

A negative prognostic value of $\geq 5 \%$ of tumor cell nuclei expressing survivin has been suggested in a group of mixed neuroendocrine tumors, mainly midgut carcinoids but also including a limited number of selected pancreatic endocrine tumors $(n=15)$ [26]. This was especially true in tumors classified as well-differentiated carcinomas, where survivin was suggested as a better prognostic marker than the Ki-67 index. No confirming reports have been published. No reports have focused on the prognostic value of survivin expression in pancreatic endocrine tumors.

In this article, we investigate the prognostic relevance of survivin in a group of 111 patients from a single institution, with information available on the Ki-67 index, WHO classification, and TNM stage.

\section{Materials and methods}

\section{Patients}

Patients treated at our clinic between 1986 and 2005 were reviewed and were included in this study if paraffin-embedded blocks of tumor tissue were available $(n=111)$. Data regarding heredity, primary tumor size, staging with computed tomography (CT), surgery, endocrine syndrome, Ki-67 index, and survival were gathered from medical records. All details concerning the material included were registered no earlier than 1986. Patient characteristics are summarized in
Table 1 Patient characteristics

\begin{tabular}{|c|c|c|}
\hline Characteristic & No. & $\%$ \\
\hline \multicolumn{3}{|l|}{ Sex } \\
\hline Female & 50 & 45 \\
\hline Male & 61 & 55 \\
\hline \multicolumn{3}{|l|}{ Tumor type } \\
\hline Nonfunctioning & 65 & 59 \\
\hline Insulinoma & 15 & 13 \\
\hline Gastrinoma & 15 & 13 \\
\hline Glucagonoma & 10 & 9 \\
\hline VIPoma & 5 & 5 \\
\hline Cushing & 1 & 1 \\
\hline \multicolumn{3}{|l|}{ Hereditary status } \\
\hline Sporadic & 89 & 80 \\
\hline MEN-1 & 21 & 19 \\
\hline VHL & 1 & 1 \\
\hline Surgery of primary tumor & 44 & 41 \\
\hline \multicolumn{3}{|l|}{ WHO classification $(n=95)$} \\
\hline Well-diff. tumor & 25 & 26 \\
\hline Well-diff. carcinoma & 63 & 66 \\
\hline Poorly diff. carcinoma & 7 & 7 \\
\hline \multicolumn{3}{|l|}{ Stage $(n=105)$} \\
\hline I & 13 & 12 \\
\hline IIa & 10 & 10 \\
\hline $\mathrm{IIb}$ & 6 & 6 \\
\hline IIIa & 2 & 2 \\
\hline IIIb & 15 & 14 \\
\hline IV & 59 & 56 \\
\hline \multicolumn{3}{|l|}{ Referred from } \\
\hline Primary uptake area & 58 & 52 \\
\hline External referral & 53 & 48 \\
\hline Size of primary tumor $(\mathrm{cm})$, median & \multicolumn{2}{|c|}{$4.6 \mathrm{~cm}$ (range $0.6-13.5)$} \\
\hline Age at diagnosis (years), median & \multicolumn{2}{|c|}{53 (range $21-86$ ) } \\
\hline
\end{tabular}

VIP vasoactive intestinal peptide, $M E N-1$ multiple endocrine neoplasia type 1, VHL von Hippel Lindau disease, WHO World Health Organization, diff. differentiated

Table 1. The median follow-up was 68 months (range 4-416 months). No patients were lost to follow-up. There was no difference in the frequency of hereditary tumor, nonfunctioning tumor, radical surgery, poorly differentiated carcinoma, or advanced stage tumor between the patients included in this study and the larger unselected material. We did, however, see a tendency toward a longer median survival $(P=0.06)$ in this patient cohort [10].

Ki-67

Data regarding the proliferation marker $\mathrm{Ki}-67$ were retrieved from patient charts. When data were not available, immunohistochemistry (IHC) for Ki-67 was 
performed at the pathology department laboratory. Paraffin-embedded sections of $4 \mu \mathrm{m}$ were used for IHC. In 14 cases, no data were attained. For antigen retrieval, sections were pretreated with 45 minutes of pressure boiling in a citrate buffer $\mathrm{pH}$ 6.0. IHC was performed using an autostainer (DakoCytomation, Carpinteria, CA, USA). Sections were incubated with an anti-Ki-67 antibody (M7240; DakoCytomation) in antibody diluent (DakoCytomation) at room temperature for $60 \mathrm{~min}$. The reaction product was revealed using Dako kit 50087 (DakoCytomation). Sections were counterstained with Mayer's hematoxylin. Initial experiments were performed with omission of the primary antibody. All sections were scored by two individuals blinded for patient outcome, according to the percentage of nuclear staining in the field with the highest percentage of staining ("hot spot"), defined after assessing the entire section (in accordance with the method applied by Professor Lars Grimelius, Department of Genetics and Pathology, Uppsala).

\section{Survivin}

Sections were deparaffinized and pretreated in TRS buffer, pH 6.0 (S1699; DakoCytomation), in a pressure cooker (Biocare Medical, Concorde, CA, USA). The staining procedure was performed in an autostainer (Autostainer Plus; DakoCytomation). A mouse monoclonal anti-survivin antibody (sc-17779; Santa Cruz Biotechnology, Santa Cruz, CA, USA) was diluted to 1:50 in antibody diluent (DakoCytomation) and incubated with sections for $1 \mathrm{~h}$ at room temperature. A Dako EnVision kit (K5007; DakoCytomation) was applied according to the manufacturer's instructions, and the chromogen 3,3'-diaminobenzidine was applied to reveal the complex. Mayer's hematoxylin was used for counterstaining. The primary antibody was omitted as the negative control in initial experiments; and human tonsil was included on a number of slides as a positive control.

Nuclear and cytoplasmic survivin expression was assessed. All sections were scored by two of the authors (S.E., M.H.L.) blinded for patient outcome. The parameter was the percentage of positive cells, and results are reported semiquantitatively as $<5 \%$ (low), 5 to $50 \%$ (medium), or $>50 \%$ of cells (high). The test-retest reliability results were calculated based on these initial assessments. The two observers reevaluated slides together when their scoring had differed.

\section{WHO classification}

Tumors were classified into three groups according to the WHO classification, based on, for example, the mitotic index and the presence of gross invasion. All cases were reevaluated based on the existing pathology, surgery, and radiology reports. In ambiguous cases, new pathology assessments were performed.

\section{TNM staging}

Staging was performed according to the suggested definitions [11]. Stage I was defined as a primary tumor confined to the pancreas and $<2 \mathrm{~cm}$. Stage IIa was a primary tumor confined to the pancreas and 2 to $4 \mathrm{~cm}$; stage IIb was a primary tumor $>4 \mathrm{~cm}$ or invading the duodenum or bile duct. Stage IIIa was defined as a tumor invading adjacent organs (stomach, spleen, colon, adrenal gland) or the wall of large vessels (celiac axis, superior mesenteric artery). The presence of lymph node metastases defined stage IIIb and distant metastases stage IV. Staging reflected the tumor burden at diagnosis and was based on data retrieved from medical records. All cases were reevaluated based on existing pathology, surgery, and radiology reports. In ambiguous cases, new pathology assessments were performed.

\section{Statistical analysis}

Test-retest reliability in the assessment of Ki-67 and survivin was assessed using the Spearman rank correlation test. The correlation between the two observers was 0.82 for the Ki-67 index score. For nuclear and cytoplasmic survivin staining, it was 0.89 and 0.84 , respectively. Differences in the expression of survivin between groups of patients were analyzed with the nonparametric KruskalWallis test.

Kaplan-Meier methodology was used to estimate survival, which was calculated as the time from diagnosis to the last date of follow-up of patients still alive at the closure of this study or death from any cause. No patients were lost to follow-up. The log-rank test was used to test differences in survival.

A multivariate Cox regression model was fitted to evaluate the independent effects of nuclear survivin, cytoplasmic survivin, and the Ki-67 index, with information on the WHO classification and TNM stage. Only 84 patients were included in this analysis owing to a missing Ki-67 index and/or WHO classification data in the remaining cases. Univariate subanalyses of patients with a well-differentiated tumor, well-differentiated carcinoma, or poorly differentiated carcinoma, respectively, were performed, as was a multivariate analysis of patients with a well-differentiated carcinoma. The proportional hazards assumption was assessed with a graphic approach. All multivariate analyses were controlled for patient age at diagnosis. A value of $P<0.05$ was considered significant. Statistical analyses were performed with the SPSS statistical package (version 15.0). 
The local ethics committee at Uppsala University approved this study, complying with the Declaration of Helsinki.

\section{Results}

\section{Survivin expression}

The frequency of nuclear and cytoplasmic survivin expression is summarized in Table 2. Most patients $(72 \%)$ had $<5 \%$ survivin-positive nuclei in their tumors, $19 \%$ had 5 to 50 , and $9 \%$ had $>50 \%$ positive nuclei (Fig. 1a). The frequency of survivin-positive cytoplasm was higher: $35 \%$ had $<5,9 \%$ had 5 to $50 \%$, and $56 \%$ had $>50 \%$ survivin-positive cytoplasm (Fig. 1b). There was a significant difference in the expression of nuclear and cytoplasmic survivin between well-differentiated tumors, well-differentiated carcinomas, and poorly differentiated carcinomas, with nuclear survivin being the most abundant in poorly differentiated carcinomas $(P<0.01)$, and cytoplasmic survivin more highly expressed in well-differentiated tumors $(P<0.01)$.

There was no significant difference in the expression of nuclear or cytoplasmic survivin between patients with functioning or nonfunctioning tumors. Tumors from patients with nonfamilial disease had a higher nuclear survivin presence $(P=0.04)$ compared to tumors from patients with multiple endocrine neoplasia type 1 (MEN-1), whereas there was a trend toward MEN-1 tumors having a higher cytoplasmic survivin presence $(P=0.08)$. However, when stratified according to the WHO classification, there were no differences in the expression of nuclear or cytoplasmic survivin between patients with sporadic or MEN-1-related tumors.

\section{Univariate survival analysis}

The presence of nuclear survivin was a negative prognostic factor in the univariate analysis (Fig. 2). Patients with $<5 \%$ positive nuclei had a median survival of 225 months [95\% confidence interval (CI) 168-281]; the corresponding figure for patients with 5 to $50 \%$ positive nuclei was 101 months (95\% CI 61-140; hazard ratio (HR) $2.4 ; P<$ $0.01]$, and for patients with $>50 \%$ positive nuclei it was 47 months (95\% CI 24-71; HR 4.9; $P<0.001)$.

There was no significant difference in survival in a three-way comparison of patients with low, medium, or high cytoplasmic survivin $(P=0.22)$. However, when dichotomizing patients at more or less than $5 \%$ cytoplasmic survivin, there was a tendency toward a longer survival in patients with high cytoplasmic survivin $(P=0.084)$ (Fig. 3). Patients with low cytoplasmic survivin lived a mean of 105 months from diagnosis (95\% CI 73-137), whereas patients with medium or high cytoplasmic survivin lived for 181 months (95\% CI 128-233). Thus, cytoplasmic survivin was certainly not a negative prognostic factor; rather, there was a tendency toward it being a positive prognostic marker.

Patients with a higher nuclear than cytoplasmic survivin score had a significantly shorter survival (50 months, 95\% CI 29-72) compared to patients with a higher cytoplasmic than nuclear survivin score (218 months, 95\% CI 157-280) or an even distribution (115 months, 95\% CI 80-151) $(P<0.001)$.

No patient with a well-differentiated tumor had high nuclear survivin expression $(>50 \%)$, and we found no difference in survival between patients with a low or medium nuclear survivin in this tumor group. Among welldifferentiated carcinomas, nuclear survivin was a borderline significant prognostic marker in the univariate analysis $(P=0.05)$. Patients with $<5 \%$ positive nuclei had a mean survival of 140 months (95\% CI 108-172). The corresponding figure for patients with 5 to $50 \%$ positive nuclei was 103 months (95\% CI 64-141), and for patients with $>50 \%$ positive nuclei it was 51 months (95\% CI 19-83). There was no significant difference in survival in this group between patients with more or less than $5 \%$ cytoplasmic survivin.
Table 2 Survivin

immunoreactivity in pancreatic endocrine tumors $(n=111)$

Total all specimens immunostained for survivin $(n=111)$. Well-differentiated tumors, Well-differentiated carcinomas, Poorly differentiated carcinomas: number of specimens immunostained for survivin when the WHO classification was available $(n=95)$

\begin{tabular}{|c|c|c|c|c|}
\hline \multirow[t]{2}{*}{ Immunoreactivity } & \multicolumn{4}{|c|}{ No. of patients } \\
\hline & Total & $\begin{array}{l}\text { Well-differentiated } \\
\text { tumors }\end{array}$ & $\begin{array}{l}\text { Well-differentiated } \\
\text { carcinomas }\end{array}$ & $\begin{array}{l}\text { Poorly differentiated } \\
\text { carcinomas }\end{array}$ \\
\hline \multicolumn{5}{|l|}{ Nuclear survivin } \\
\hline$<5 \%$ & $80(72 \%)$ & $23(92 \%)$ & $44(70 \%)$ & $2(29 \%)$ \\
\hline $5-50 \%$ & $21(19 \%)$ & $2(8 \%)$ & $12(19 \%)$ & $3(43 \%)$ \\
\hline$>50 \%$ & $10(9 \%)$ & - & $7(11 \%)$ & $2(29 \%)$ \\
\hline \multicolumn{5}{|c|}{ Cytoplasmic survivin } \\
\hline$<5 \%$ & $39(35 \%)$ & $4(16 \%)$ & $28(44 \%)$ & $3(43 \%)$ \\
\hline $5-50 \%$ & $10(9 \%)$ & - & $6(10 \%)$ & $1(14 \%)$ \\
\hline$>50 \%$ & $62(56 \%)$ & $21(84 \%)$ & $29(46 \%)$ & $3(33 \%)$ \\
\hline
\end{tabular}



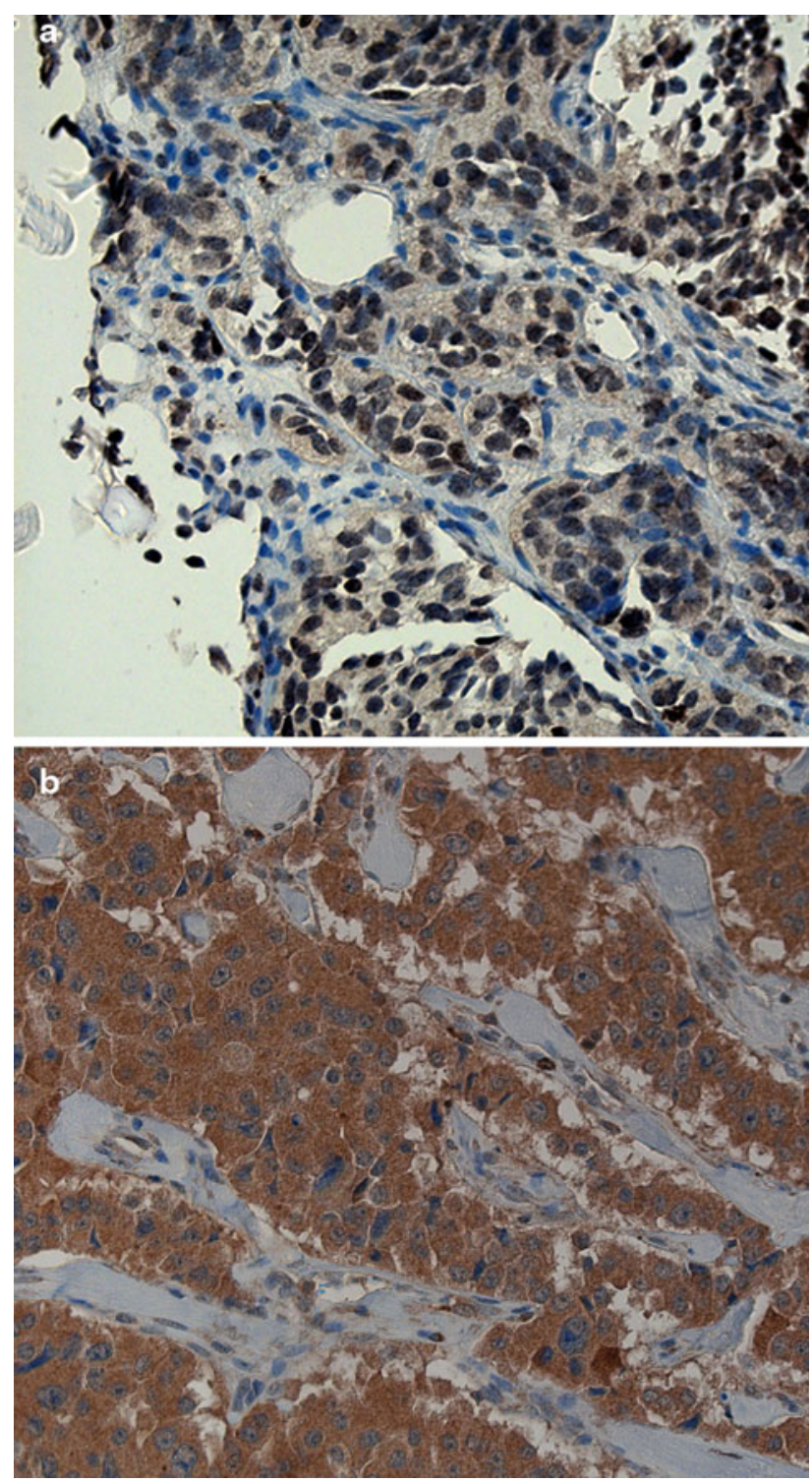

Fig. 1 a Pancreatic endocrine tumor with a high expression of nuclear survivin. Dark brown immunoreactivity shows survivinexpressing tumor cell nuclei. Surrounding fibroblast cell nuclei lack survivin and are blue. b Pancreatic endocrine tumor with a low expression of nuclear survivin and abundant expression of cytoplasmic survivin, as indicated by the brown chromogen. Surrounding fibroblast cells lack survivin expression

Among patients with well-differentiated carcinomas and a Ki-67 index $\geq 2 \%$, having a nuclear survivin level of $>5 \%$ showed a tendency toward being a significant negative prognostic marker $(P=0.08)$, and a cutoff of $<50 \%$ or $>50 \%$ rendered a highly significant difference in survival $(P<0.001)$ (Fig. 4). Patients with a high nuclear survivin and a Ki-67 index $\geq 2 \%$ lived, on average, 21 months from diagnosis (95\% CI 14-29), whereas the corresponding figure for patients with a low or medium nuclear survivin level was 99 months (95\% CI 75-123).

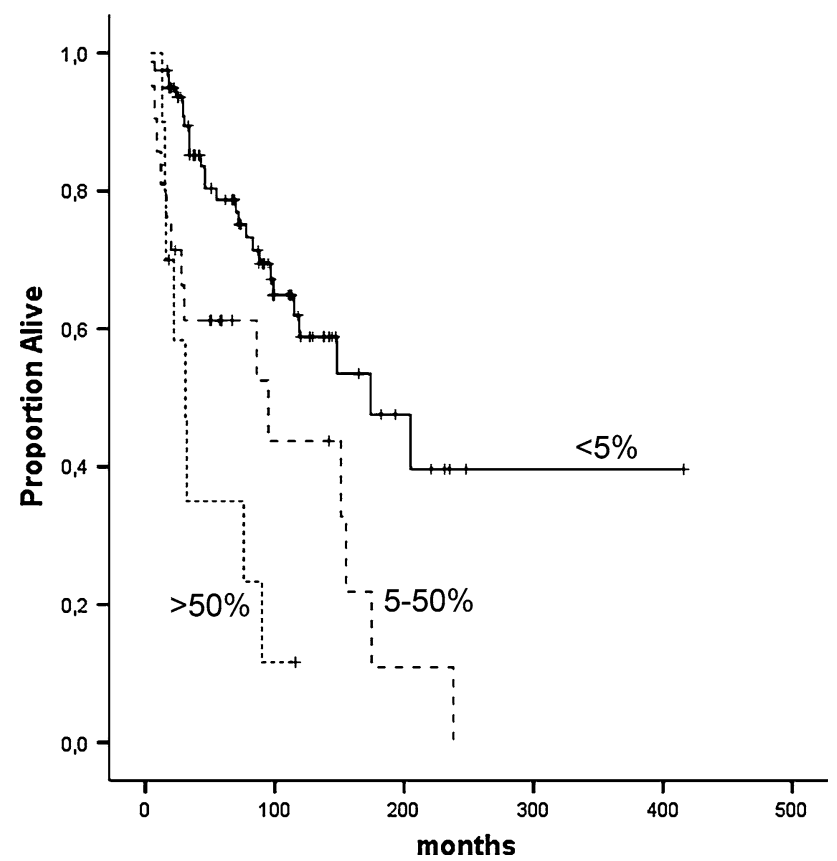

Fig. 2 High nuclear survivin is a significant negative predictor of survival $(P<0.001)$

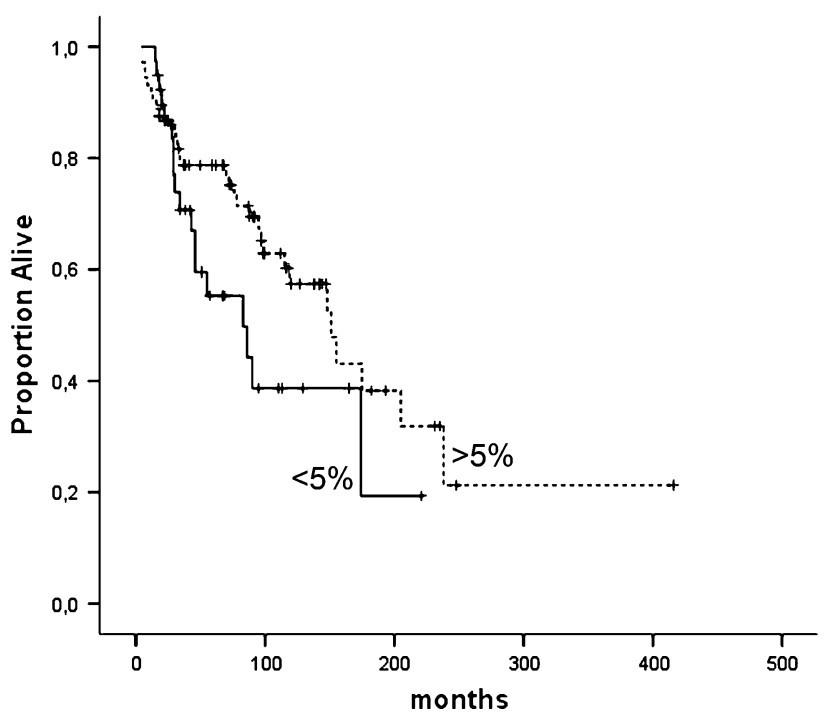

Fig. 3 Tendency toward cytoplasmic survivin being a positive predictor of survival $(P=0.084)$

In patients with poorly differentiated carcinoma, a nuclear survivin presence of $<5 \%$ emerged as a positive prognostic marker $(P=0.04)$. Patients with a low nuclear survivin presence, on average, lived twice as long from diagnosis, 40 months (95\% CI 28-52), compared to 22 months (95\% CI 12-31) for patients with a medium or high nuclear survivin presence. 


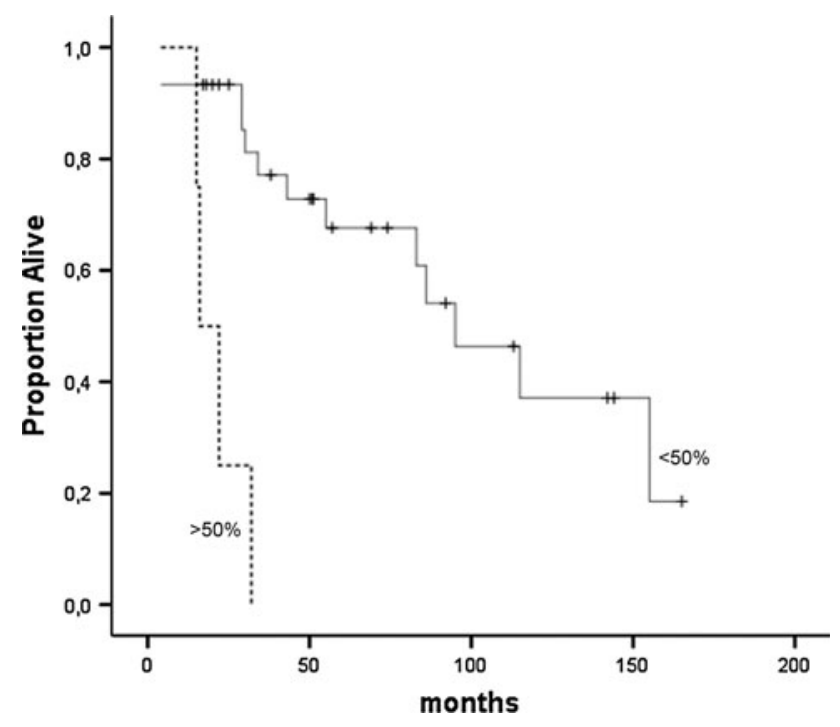

Fig. 4 Among patients with well-differentiated carcinoma and Ki$67>2 \%$, patients with a nuclear survivin $>50 \%$ fared significantly worse

Multivariate survival analysis

When controlled for the Ki-67 index, cytoplasmic survivin, WHO classification, and TNM stage in a multivariate model, high nuclear survivin emerged as a significant negative prognostic marker with an HR of $5.7(P<0.01)$. A medium nuclear survivin presence carried an HR of 1.8 but was not significant $(P=0.21)$. High cytoplasmic survivin was not a significant prognostic factor (HR 0.94; $P=0.90)$.

In a multivariate analysis that included only patients with well-differentiated carcinoma and that evaluated nuclear survivin, cytoplasmic survivin, and the Ki-67 index, only high nuclear survivin emerged as a significant prognostic marker (HR 3.8; $P=0.03$ ).

\section{Discussion}

Predicting the prognosis for the individual cancer patient is of paramount importance. The factors used in this tumor group today are not satisfactory, and there is a need for additional markers to fine-tune this process. We report a prognostic value of survivin immunohistochemistry in a large group of patients with pancreatic endocrine tumors treated at a single institution. A prognostic value of survivin has previously been suggested in a study of 15 pancreatic endocrine tumors [26]. We present confirmatory data on a more comprehensive group of 111 patients.

This was also a retrospective study, with the limitations that it implies. A prospective confirmation of our results would of course provide a stronger foundation for clinical application. The patients evaluated in this study constitute a selection of all patients treated at our clinic between 1986 and 2005 based on tumor tissue availability. Although we found no differences in tumor stage or WHO classification between included and not included patients, we did see a tendency toward a longer survival among patients selected for this study.

The assessment of survivin expression was done in a semiquantitative manner. Although a quantitative analysis provides more detailed data, it causes a loss of simplicity and clinical applicability. Semiquantitative analysis of survivin immunoreactivity is simple and straightforward, and it can easily be used in a clinical setting, which is a major advantage.

We found a highly significant negative prognostic value of nuclear survivin. Patients with low nuclear survivin lived almost five times longer than those with high nuclear survivin and twice as long as patients with medium high nuclear survivin. The prognostic value of high nuclear survivin was confirmed when controlled for the WHO classification, TNM stage, and Ki-67 index in a multivariate analysism; and it can thus be called an independent prognostic marker. In a multivariate analysis, a Ki-67 index of more than $2 \%$ did not significantly affect survival time. It thus seems that nuclear survivin is at least as good a marker for poor prognosis as a cutoff of the Ki-67 index at $\geq 2 \%$. In addition, semiquantitative assessment of nuclear survivin is relatively straightforward compared to the tedious process of calculating a Ki-67 index.

The value of cytoplasmic survivin expression was less clear. We did see a tendency toward patients with a low cytoplasmic survivin having a shorter survival. Although this was not significant and did not persist in the multivariate analysis, it is an interesting finding. We also found that patients with a predominantly cytoplasmic survivin do much better than patients with predominantly nuclear survivin. The former lived, on average, four times longer from the time of the diagnosis. Thus, it seems less favorable to have survivin in the tumor cell nuclei than in the cytoplasm. The reason for this is not entirely clear. Different functions of survivin in the nucleus and cytoplasm have been suggested, and different splice variants of survivin are known to locate in different subcellular compartments [2729]. It has been suggested that alternative survivin splice variants could be more relevant for prognostication. However, others report that these alternative splice variants do not participate in cell division [30].

There was a marked difference in the expression of both nuclear and cytoplasmic survivin between well-differentiated tumors, well-differentiated carcinomas, and poorly differentiated carcinomas. Well-differentiated tumors, the group with the most favorable prognosis, in most cases had very low nuclear and high cytoplasmic survivin. Thus, with 
the semiquantitative evaluation of survivin used here, no prognostic significance could be seen in this tumor group.

Among well-differentiated carcinomas, it is often exceedingly difficult to make an accurate prediction of prognosis for the patients: Some patients have indolent tumors, whereas others experience rapid tumor progression. A Ki-67 index of $\geq 2 \%$ is often cited as a negative sign in these patients. High nuclear survivin expression was a bit more common in this group, although tumors with low survivin still dominated. High nuclear survivin was an independent sign of a poor prognosis: When looking only at patients with well-differentiated carcinoma and a Ki-67 index of $\geq 2 \%$, there was a marked, almost fivefold, difference in survival from time of diagnosis between patients with a nuclear survivin level over or under $50 \%$ (Fig. 4). Thus, nuclear survivin could have important prognostic implications in the subgroup of patients with well-differentiated carcinoma and a Ki-67 index of $\geq 2 \%$. Patients with low or medium nuclear survivin did indeed have a lower mean Ki-67 index (Table 3). However, because the standard deviation of the Ki-67 index was so great, prediction based on this marker is difficult; thus, nuclear survivin could be an important additional marker helping to predict survival and decisions regarding the treatment approach.

Poorly differentiated carcinomas are aggressive cancers with a generally poor prognosis. However, even in this group survival varies, and some patients fare notably better than others. In this study, we found a doubled survival time in patients with low nuclear survivin compared to patients with medium or high survivin. Although survival is still relatively short, this is a marked difference with potentially important consequences for the individual patients.

Grabowski et al. suggested a prognostic value of nuclear survivin in material consisting of different types of neuroendocrine gastrointestinal tumors [26]. Our data support their observations and unequivocally demonstrate a prognostic value of nuclear survivin in pancreatic endocrine tumors. This was especially true for patients with well-

Table $3 \mathrm{Ki}-67$ index in relation to nuclear survivin expression

\begin{tabular}{cclll}
\hline $\begin{array}{l}\text { Nuclear } \\
\text { survivin }\end{array}$ & \multicolumn{3}{l}{ KI-67 index (mean and SD) } & \\
\cline { 2 - 5 } & Total & $\begin{array}{l}\text { Well- } \\
\text { differentiated } \\
\text { tumors }\end{array}$ & $\begin{array}{l}\text { Well- } \\
\text { differentiated } \\
\text { carcinomas }\end{array}$ & $\begin{array}{l}\text { Poorly } \\
\text { differentiated } \\
\text { carcinomas }\end{array}$ \\
\hline$<5 \%$ & $4.3(5.8)$ & $2.1(2.6)$ & $4.7(5.8)$ & $18(18)$ \\
$5-50 \%$ & $14(17)$ & 0 & $6.8(8.3)$ & $37(15)$ \\
$>50 \%$ & $16(18)$ & - & $11(14)$ & $41(15)$ \\
\hline
\end{tabular}

Total average Ki-67 index in all WHO classes, with regard to expression of nuclear survivin. In the next three columns are indexes for the three WHO classes $(n=84)$ differentiated carcinoma, where a prognosis is notoriously difficult to predict. Being relatively simple to evaluate and adding significant prognostic value, survivin could prove an important addition to the limited arsenal of tools available for prognostic guidance in these patients. We hope to see further confirmatory studies and subsequent incorporation of survivin evaluation in the clinical routine for these patients.

Acknowledgments The authors thank Professor Lars Grimelius for expert pathology advice and Ulrika Larsson for technical assistance.

Grant support Britt Skogseid was supported by the Swedish Cancer Society, Swedish Research Council, and Lion's Fund for Cancer Research.

Open Access This article is distributed under the terms of the Creative Commons Attribution Noncommercial License which permits any noncommercial use, distribution, and reproduction in any medium, provided the original author(s) and source are credited.

\section{References}

1. Hochwald SN, Zee S, Conlon KC et al (2002) Prognostic factors in pancreatic endocrine neoplasms: an analysis of 136 cases with a proposal for low-grade and intermediate-grade groups. J Clin Oncol 20:2633-2642

2. Tomassetti P, Campana D, Piscitelli L et al (2005) Endocrine pancreatic tumors: factors correlated with survival. Ann Oncol 16:1806-1810

3. Pape UF, Bohmig M, Berndt U et al (2004) Survival and clinical outcome of patients with neuroendocrine tumors of the gastroenteropancreatic tract in a German referral center. Ann N Y Acad Sci 1014:222-233

4. Panzuto F, Nasoni S, Falconi M et al (2005) Prognostic factors and survival in endocrine tumor patients: comparison between gastrointestinal and pancreatic localization. Endocr Relat Cancer 12:1083-1092

5. Kloppel G, Perren A, Heitz PU (2004) The gastroenteropancreatic neuroendocrine cell system and its tumors: the WHO classification. Ann N Y Acad Sci 1014:13-27

6. Pelosi G, Bresaola E, Bogina G et al (1996) Endocrine tumors of the pancreas: $\mathrm{Ki}-67$ immunoreactivity on paraffin sections is an independent predictor for malignancy: a comparative study with proliferating-cell nuclear antigen and progesterone receptor protein immunostaining, mitotic index, and other clinicopathologic variables. Hum Pathol 27:1124-1134

7. Clarke MR, Baker EE, Weyant RJ et al (1997) Proliferative activity in pancreatic endocrine tumors: association with function, metastases, and survival. Endocr Pathol 8:181-187

8. La Rosa S, Sessa F, Capella C et al (1996) Prognostic criteria in nonfunctioning pancreatic endocrine tumours. Virchows Arch 429:323-333

9. Deschamps L, Handra-Luca A, O'Toole D et al (2006) CD10 expression in pancreatic endocrine tumors: correlation with prognostic factors and survival. Hum Pathol 37:802-808

10. Ekeblad S, Skogseid B, Dunder K et al (2008) Prognostic factors and survival in 324 patients with pancreatic endocrine tumor treated at a single institution. Clin Cancer Res 14:7798-7803

11. Rindi G, Kloppel G, Alhman H et al (2006) TNM staging of foregut (neuro)endocrine tumors: a consensus proposal including a grading system. Virchows Arch 449:395-401 
12. Fischer L, Kleeff J, Esposito I et al (2008) Clinical outcome and long-term survival in 118 consecutive patients with neuroendocrine tumours of the pancreas. Br J Surg 95:627-635

13. LaCasse EC, Baird S, Korneluk RG et al (1998) The inhibitors of apoptosis (IAPs) and their emerging role in cancer. Oncogene 17:3247-3259

14. Deveraux QL, Reed JC (1999) IAP family proteins: suppressors of apoptosis. Genes Dev 13:239-252

15. Marusawa H, Matsuzawa S, Welsh K et al (2003) HBXIP functions as a cofactor of survivin in apoptosis suppression. EMBO J 22:2729-2740

16. Altieri DC (2006) The case for survivin as a regulator of microtubule dynamics and cell-death decisions. Curr Opin Cell Biol 18:609-615

17. Lens SM, Vader G, Medema RH (2006) The case for survivin as mitotic regulator. Curr Opin Cell Biol 18:616-622

18. Velculescu VE, Madden SL, Zhang L et al (1999) Analysis of human transcriptomes. Nat Genet 23:387-388

19. Pennati M, Folini M, Zaffaroni N (2008) Targeting survivin in cancer therapy. Expert Opin Ther Targets 12:463-476

20. Kelly RJ, Lopez-Chavez A, Citrin D et al (2011) Impacting tumor cell-fate by targeting the inhibitor of apoptosis protein survivin. Mol Cancer 10:35

21. Lu B, Gonzalez A, Massion PP et al (2004) Nuclear survivin as a biomarker for non-small-cell lung cancer. Br J Cancer 91:537540

22. Fields AC, Cotsonis G, Sexton D et al (2004) Survivin expression in hepatocellular carcinoma: correlation with proliferation, prognostic parameters, and outcome. Mod Pathol 17:1378-1385
23. Okada E, Murai Y, Matsui K et al (2001) Survivin expression in tumor cell nuclei is predictive of a favorable prognosis in gastric cancer patients. Cancer Lett 163:109-116

24. Kennedy SM, O’Driscoll L, Purcell R et al (2003) Prognostic importance of survivin in breast cancer. $\mathrm{Br} \mathrm{J}$ Cancer 88: 1077-1083

25. Tonini G, Vincenzi B, Santini D et al (2005) Nuclear and cytoplasmic expression of survivin in 67 surgically resected pancreatic cancer patients. Br J Cancer 92:2225-2232

26. Grabowski P, Griss S, Arnold CN et al (2005) Nuclear survivin is a powerful novel prognostic marker in gastroenteropancreatic neuroendocrine tumor disease. Neuroendocrinology 81:1-9

27. Mahotka C, Liebmann J, Wenzel M et al (2002) Differential subcellular localization of functionally divergent survivin splice variants. Cell Death Differ 9:1334-1342

28. Caldas H, Jiang Y, Holloway MP et al (2005) Survivin splice variants regulate the balance between proliferation and cell death. Oncogene 24:1994-2007

29. Badran A, Yoshida A, Ishikawa K et al (2004) Identification of a novel splice variant of the human anti-apoptopsis gene survivin. Biochem Biophys Res Commun 314:902-907

30. Noton EA, Colnaghi R, Tate S et al (2006) Molecular analysis of survivin isoforms: evidence that alternatively spliced variants do not play a role in mitosis. J Biol Chem 281:1286-1295 